\title{
Galling insects are bioindicators of environmental quality in a Conservation Unit
}

André Portugal Santana ${ }^{1,2}$ and Rosy Mary dos Santos Isaias ${ }^{1^{*}}$

Received: January 29, 2014. Accepted: June 10, 2014

\begin{abstract}
Galls are well distributed across the World and among plant families. Their diversity can support the status of conservation of an area as an urban park, once inventories are presented. These inventories also help to understand the morphological patterns of the galls, based on their most common shape, color, host botanical families, inducers and galled organs. This study is about an inventory of galls at Parque Estadual Serra Verde, Brazil. This conservation unit is an urban park strongly anthropized in a transition area of Cerrado and Mata Atlântica. Galls from four different trails were observed, and collected monthly during one year. The terminology morphospecies was used to distinguish the galls because the identification of the inducers were not always possible. Seventy five morphospecies of galls belonging to 43 host plant species of 24 botanical families were observed. Mostly of the galls was induced by Diptera:Cecidomyiidae, in Fabaceae and Myrtaceae. The most common traits were the globoid shape and green color. The leaves were the most frequent galled organ and followed by the stems. All these tendencies had been already observed in other inventories. Comparing current results with other studies at similar areas, we can assume that the Parque Estadual Serra Verde is very important for conservation. Urban green areas are subject to high disturbance and degradation but also increase the quality of life for the population inhabiting the areas nearby. The diversity of galls at Parque Estadual Serra Verde reflects an area with high levels of stress but with moderate botanical diversity. These features make this protected area an important site for the continuous conservation and regeneration, and highlight the environmental value of Parque Estadual Serra Verde.
\end{abstract}

Keys words: Biodiversity, conservation unit, galling insects, inventory, neotropical galls.

\section{Introduction}

Galls are abnormal growths of plant tissues induced by several living organisms that maintain an intimate endophytic interaction with their host plants and organs (Mani 1964, Dreger-Jauffret \& Shorthouse 1992). The galls can be induced by virus, bacteria, fungi, nematodes, mites, and mainly by insects, which are called entomogenous galls. These galls are distributed across wide biogeographical areas and among plant families (Fernandes \& Martins 1985, Arduin \& Kraus 2001). Based on the growing diversity of their inducers and the correlation between the number of galls and plant richness, Espírito-Santo \& Fernandes (2007) estimated the existence of about 133,000 of galling insect species in the World. Among the botanical families, the most affected by this kind of interactions are the Fabaceae, Asteraceae, Myrtaceae, Malpighiaceae and Melastomataceae (Fernandes et al. 1997, Gonçalves-Alvim \& Fernandes 2001, Maia \& Fernandes 2004).

Gall inducers are poorly known taxonomically, especially in the Neotropics, where most of the species are new to science. Consequently, expressions such as morphospecies or gall morphotypes are proposed and widely used based on their external morphology in combination with the host organ or host plant species (Price et al. 1998, Carneiro et al. 2009, Costa, Fagundes \& Neves 2010). Among galling insects' families, the most prominent is the Cecidomyiidae (Diptera), which is a highly specialized group with the galling habit, and Cynipidae (Hymenoptera). Moreover, some species of Coleoptera, Lepidoptera, Hemiptera and Thysanoptera are also reported as gall inducers (Fernandes et al. 1997, Araújo et al. 2007, Santos et al. 2010).

These insects are excellent models for studies on the ecological diversity and specificity due to their richness, abundance and sessile habit. They are easily collected, and have predictable responses to environmental changes, as well as possess a high specificity to their host plants (Fernandes et al. 2009). Apart from the gall inducers, other organisms may also be involved in the galling microenvironment such as the parasitoids, the predators and the inquilines (Maia \& Fernandes 2004). These natural enemies coupled with the high diversity and characteristics

\footnotetext{
${ }^{1}$ Universidade Federal de Minas Gerais, ICB/Departamento de Botânica. Av. Presidente Antônio Carlos, 6627, Caixa Postal 486 - Pampulha - Belo Horizonte - MG - Brasil CEP: 31270-901 Telefone: +55 31 3409.2687.

${ }^{2}$ Instituto Estadual de Florestas. Diretoria de áreas protegidas. Rodovia Pref. Américo Gianetti, 4143 - Edifício Minas - Belo Horizonte, MG

* Autor para correspondência: rosy@icb.ufmg.br
} 
of the host plants (chemical, physiological, anatomical, genetic), climatic conditions of the habitat, anthropogenic disturbances (urbanization), and biogeographic gradients (elevation, latitude, temperature and humidity) are the main factors that determine the diversity and distribution of galling herbivores (Fernandes 1992, Lara \& Fernandes 1996, Fernandes et al. 1997).

Currently, the effect of habitat fragmentation over the species composition must be considered, and has been widely investigated. Environments subject to greater nutritional and hygrothermal stresses are richer in species of galls (Fernandes \& Price 1992). In addition, species composition and richness of galls are directly related to the local flora (Resende et al. 2002, Carneiro et al. 2009). The gall inducers are also good indicators of environmental conditions and biodiversity, especially where there is a mosaic of habitats established by water, heat and anthropogenic stresses (Fernandes \& Price 1988, 1992, Fernandes et al. 1995, Resende et al. 2002, Moreira et al. 2007).

Lately, studies of galls in conservation units such as parks are being carried out in greater numbers. This is the case of Estação Ecológica de Pirapitinga (Gonçalves-Alvim \& Fernandes 2001), Parque Nacional da Serra do Cipó (Lara \& Fernandes 1996, Coelho et al. 2009), Área de Proteção Ambiental da Serra de São José (Maia \& Fernandes 2004), Reserva Particular do Patrimônio Natural Fazenda Bulcão (Fernandes \& Negreiros 2006), Parque Nacional Vale do Catimbau (Silva et al. 2011), Refúgio Ecológico Charles Darwin (Santos-Mendonça et al. 2007), Parque Estadual Pireneus (Araújo et al. 2007), Reserva Biológica Estadual da Praia do Sul (Maia \& Oliveira, 2010), and a range of conservation units within the Serra do Espinhaço (Carneiro et al. 2009). In spite of the large amount of the academic work that has being carried out in this field, much remains to be done, regarding the ecology and biology of the organisms involved in galling habit.

Most of these conservation units are located in rural regions, where urbanization initiatives have had little impact. However, there are some urban parks that have important conservation roles due to the fragmentation of natural vegetation covering, and consequently loss of life quality. In contrast, urban areas are characterized by intense human activity, strong presence of residential, industrial, and commercial centers and vestiges of native and/or modified vegetation. Thus, the study of the diversity of remaining organisms in these areas would provide good bioindicators of environmental quality. This would strongly apply to the galling insects, for they have a great sensitivity to environmental changes, and a significant decrease of diversity has been observed, as urbanization increases (Julião et al. 2005).

How the green areas in urban regions are managed can greatly affect their species composition. The improvement of knowledge about the organisms, and of their interactions may reinforce the importance of green areas for the cities, as they are good indicators of quality of life in an urban setup. Herein, we carried out an inventory on the diversity of galls of Parque Estadual Serra Verde as the first step to demonstrate the conservation status of the area, and to set this urban park as a reference for scientific research and environmental interpretation for the benefit of the community of Belo Horizonte city. We also compare and contrast our data against other academic works searching for existing patterns within the main groups of host plants and gall inducers, identifying their main characteristics and detailing their diversity. It is proposed that these indicators will provide us with an effective benchmark regarding the conservation status of the Parque Estadual Serra Verde.

\section{Material and Methods}

\section{Study Area}

This research was carried out at the Parque Estadual Serra Verde (Serra Verde State Park) - henceforth PESV $\left(19^{\circ} 47^{\prime} 17.41^{\prime \prime} S\right.$ and $\left.43^{\circ} 57^{\prime} 31.52^{\prime \prime} \mathrm{W}\right)$, a conservation unit of 142 hectares located in Belo Horizonte municipality, Minas Gerais State, Brazil. The PESV was created by a State Decree of December 12th, 2007, and its area was augmented by the State Decree number 45,077 of March 30th, 2009, when it reached its present dimension. Since its inception, it has been managed by the Instituto Estadual de Florestas de Minas Gerais (IEF-MG). It is a transition ecoregion located between the threatened biomes of the Cerrado and the Mata Atlântica. It is strongly anthropized, except for some small spots where there are remnants of the original forest. Due to its location within an urban area, the park has suffered historical pressures from the subsequent urban sprawl and a high population growth. Its climate is classified as semihumid with a dry season (Awi) from April to September. The annual mean temperature ranges from $15^{\circ} \mathrm{C}$ to $21.1^{\circ} \mathrm{C}$; the rates of annual precipitation range from $190 \mathrm{~mm}$ to 1,515 $\mathrm{mm}$, and the main soil types are red-yellow argisols and haplic cambisols, poor in nutrients for the plants (IEF 2010).

The PESV has several trails as detailed in its Management Plan in 2010. Four of these trails have been used for educational and recreational purposes by the community and the Park staff for environmental education. They are: the Trilha da Mata (considered in the management plan as part of the Trilha da Macaúba), the Trilha do Estacionamento (considered in the management plan as part of the Trilha da Comunidade), the Trilha do Pica-Pau, and the Trilha do Curiango (Fig. 1). All together, the length of these trails totals approximately two kilometers.

\section{Data sampling}

The richness and seasonality of gall morphospecies were surveyed once a month from September 2012 to August 2013. Each field survey lasted six hours for the four trails, amounting to 72 hours of observation. We took into 


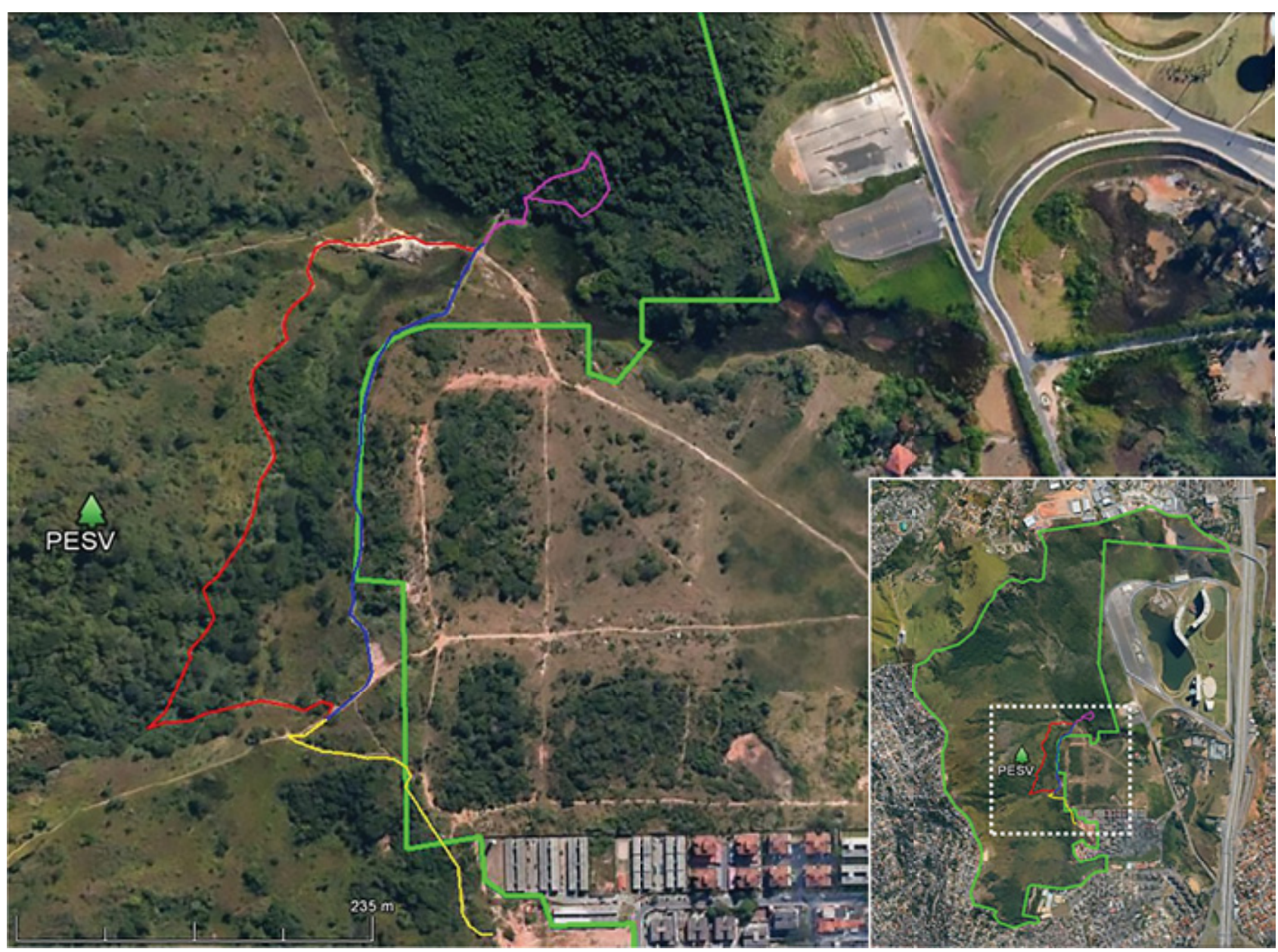

Figure 1. Ecological trails of PESV: the Trilha do Curiango (red), the Trilha do Pica-Pau (blue), the Trilha da Mata (pink); the Trilha do Estacionamento (yellow); park boundaries (green). Extracted from Google Earth ${ }^{\circ}$.

consideration the gall shape/morphotype, the host plant and the inducer in order to classify the different morphospecies.

All gall morphospecies observed in the vegetation (epigeal part) up to two meters high received a temporary identification tag and were photographed. A sample of each gall morphospecies was then collected and placed in plastic bags for further identification and description in the laboratory. At the time of collection, data related to the morphospecies, their host species, and habitat were recorded. The categorization of the morphotypes and the descriptive items proposed by Isaias et al. (2013) were adopted. In the lab, part of the samples was packed in transparent plastic pots in an attempt to rear adult insects. Another part of the samples was sectioned under a stereomicroscope to gain access to the inducer as well as to describe some gall features. All inducers were preserved in $70 \%$ ethanol and some of them were sent to specialists for identification. Furthermore, literature already mentioned in this article, especially the surveys, was also employed in the identification process.

Fertile branches of the host plants were used on the identification of the plants, and were deposited in the Her- barium BHCB (169399 to 169428). The classification of the host angiosperms followed the proposal of the Angiosperm Phylogeny Group III - APGIII (Chase \& Reveal 2009).

\section{Results}

The 75 morphospecies of galls (Fig. 2-6) observed at the PESV belong to 43 host plant species of 24 botanical families (Table 1). The most common host plants belong to the Fabaceae $(29 \%)$ with 22 morphospecies of galls, whose host plants belong to 10 species of eight genera, and Myrtaceae (13\%) with 10 morphospecies of galls, whose host plants belong to five species of four genera (Fig. 7). Among the associated guild found in the galls, there was a predominance of Diptera: Cecidomyiidae (40\%), Hymenoptera (17\%), and Thysanoptera (11\%) (Fig. 08). The months with the highest presence of the morphospecies were June with 57 morphospecies (76\%), followed by May and October with 52 (69\%) and 49 (65\%) different morphospecies, respectively. The months with less than $50 \%$ of the morphospecies were September, January and March, when a homogeneous temporal distribution 

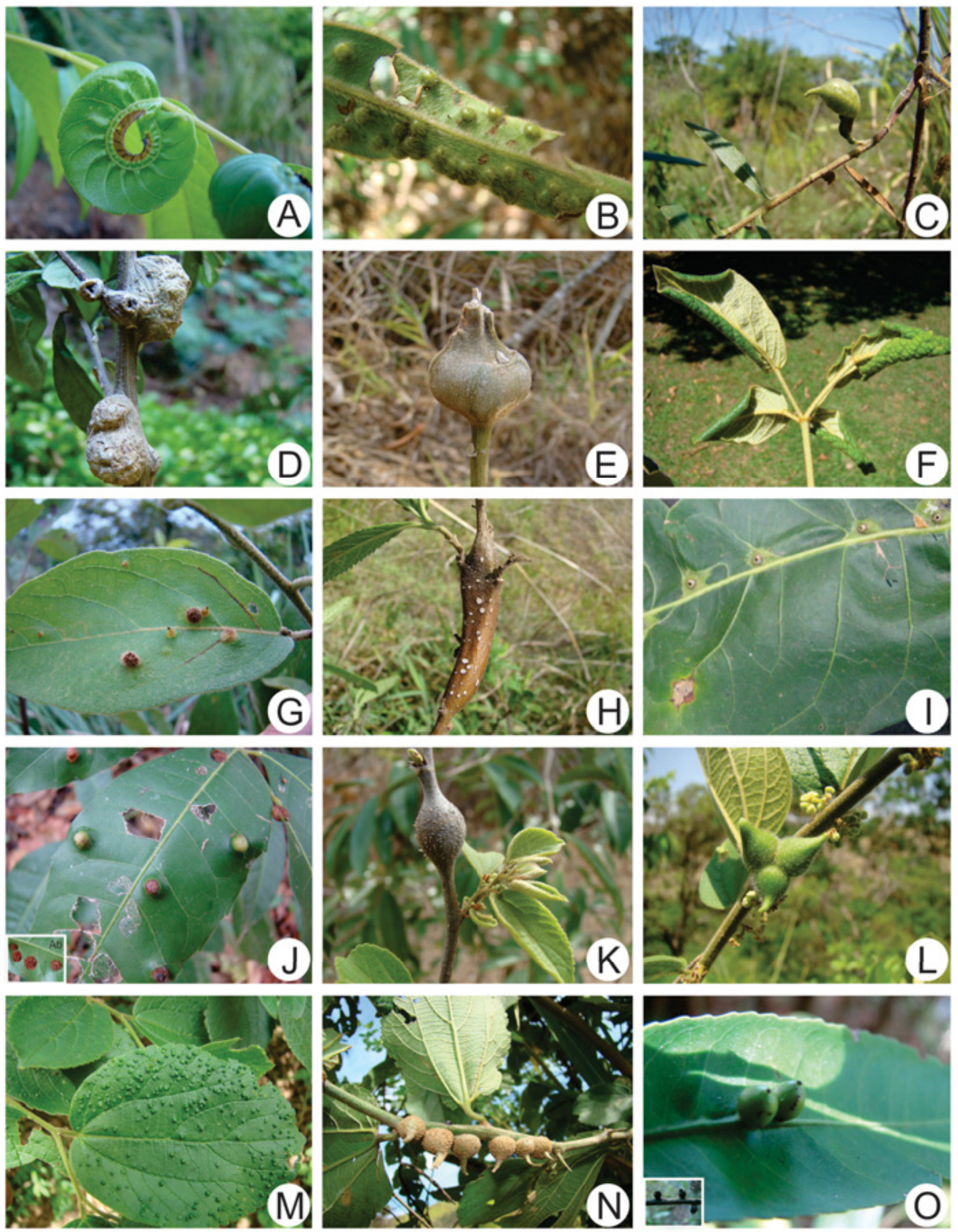

Figure 2. Morphospecies of galls of the ecological trails of the PESV. A) Astronium fraxinifolium (Anacardiaceae), B) Guatteria sellowiana (Anonaceae), C) Baccharis dracunculifolia (Asteraceae), D-E) Vernonantura phosphorica (Asteraceae), F) Handroanthus ochraceus (Bignoniaceae), G) Cordia sellowiana (Boraginaceae), H) Cordia verbenacia (Boraginaceae), I-J) Protium heptaphyllum (Burseraceae), K-N) Celtis pubescens (Cannabaceae), O) Maytenus salicifolia (Celastraceae). Ad - adaxial side; St - stem. 

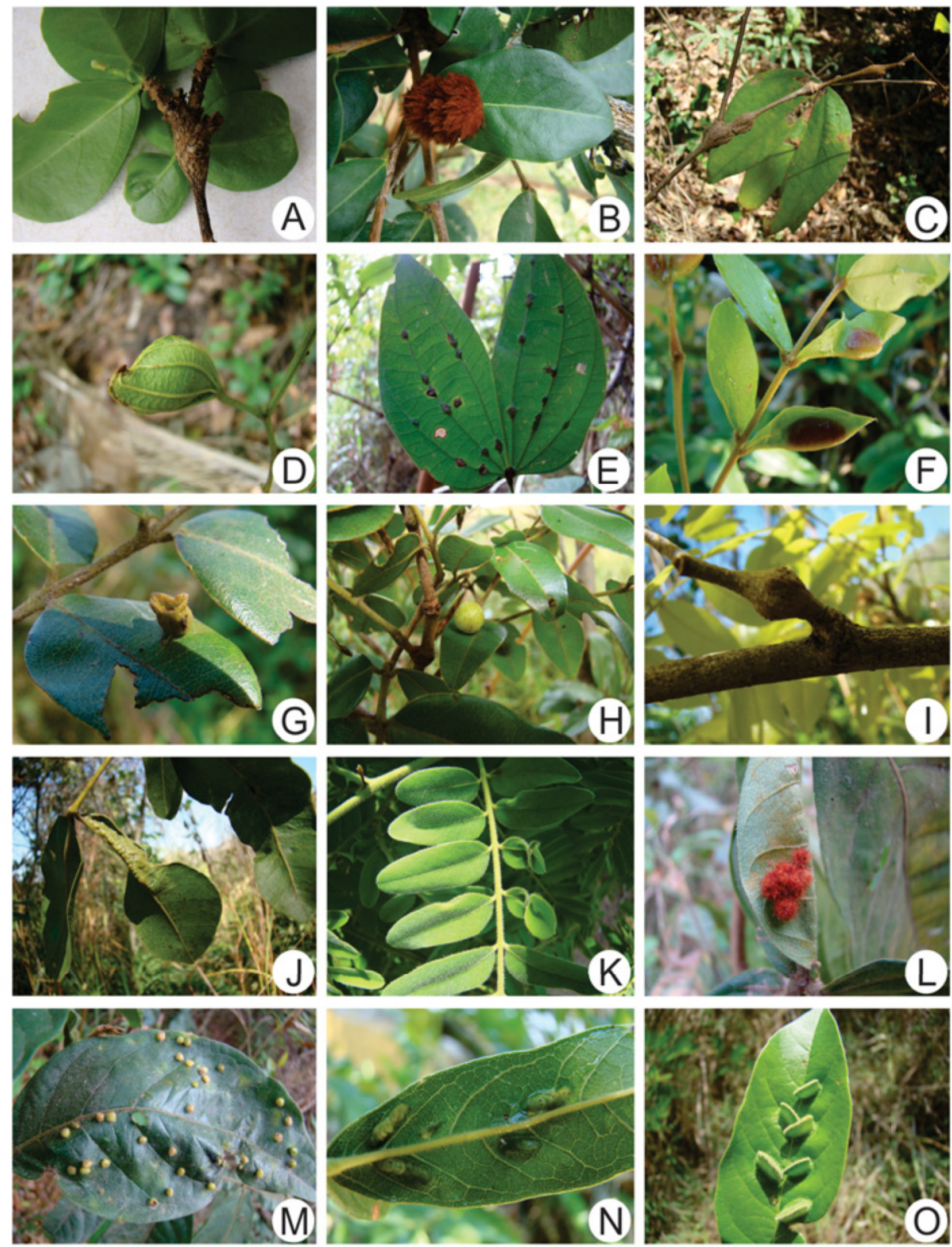

Figure 3. Morphospecies of galls of the ecological trails of the PESV. A) Erythroxylum cuneifolium (Erythroxylaceae), B) Erythroxylum suberosum (Erythroxylaceae), C-E) Bauhinia ungulata (Fabaceae), F-J) Copaifera langsdorffii (Fabaceae), K) Dalbergia villosa (Fabaceae), L-M) Inga ingoides (Fabaceae), N) Lonchocarpus cultratus (Fabaceae), O) Lonchocarpus muehlbergianus (Fabaceae). 

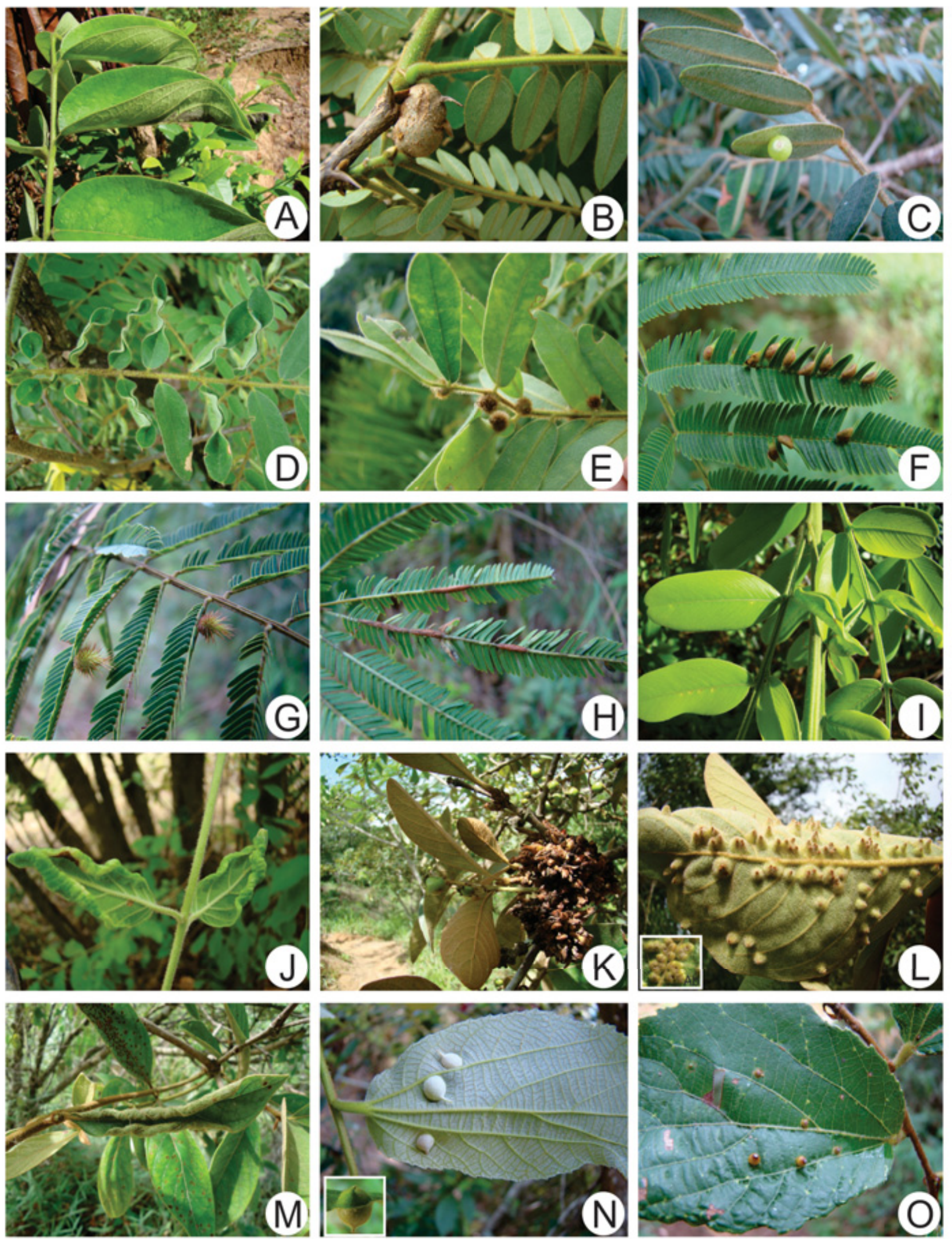

Figure 4. Morphospecies of galls of the ecological trails of the PESV. A) Machaerium dimorphandrum (Fabaceae), B-E) Machaerium nyctitans (Fabaceae), F-H) Piptadenia gonoacantha (Fabaceae), I) Platypodium ellegans (Fabaceae); J) Nectandra oppositifolia (Lauraceae), K-M) Byrsonima sp. (Malpighiaceae), N-O) Luehea candicans (Malvaceae). Ad - adaxial side. 

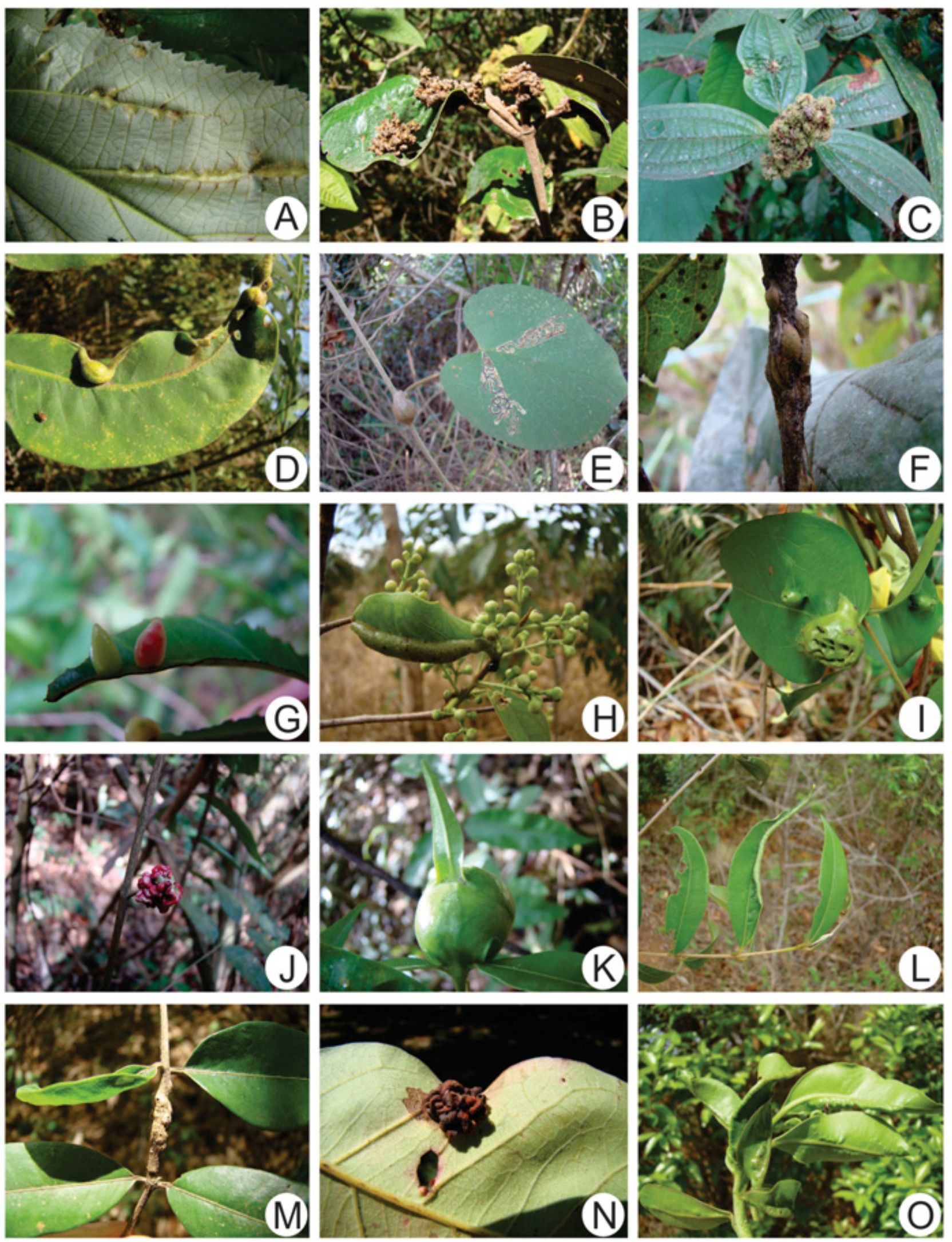

Figure 5. Morphospecies of galls of the ecological trails of the PESV. A) Luehea candicans (Malvaceae), B) Miconia albicans (Melastomataceae), C) Miconia ibaguensis (Melastomataceae), D) Trichilia lepidota (Meliaceae), E) Abuta sp (Menispermaceae), F) Campomanesia sp. (Myrtaceae), G-I) Eugenia florida (Myrtaceae), J-M) Myrcia splendens (Myrtaceae), N) Myrcia tomentosa (Myrtaceae), O) Myrciaria floribunda (Myrtaceae). 

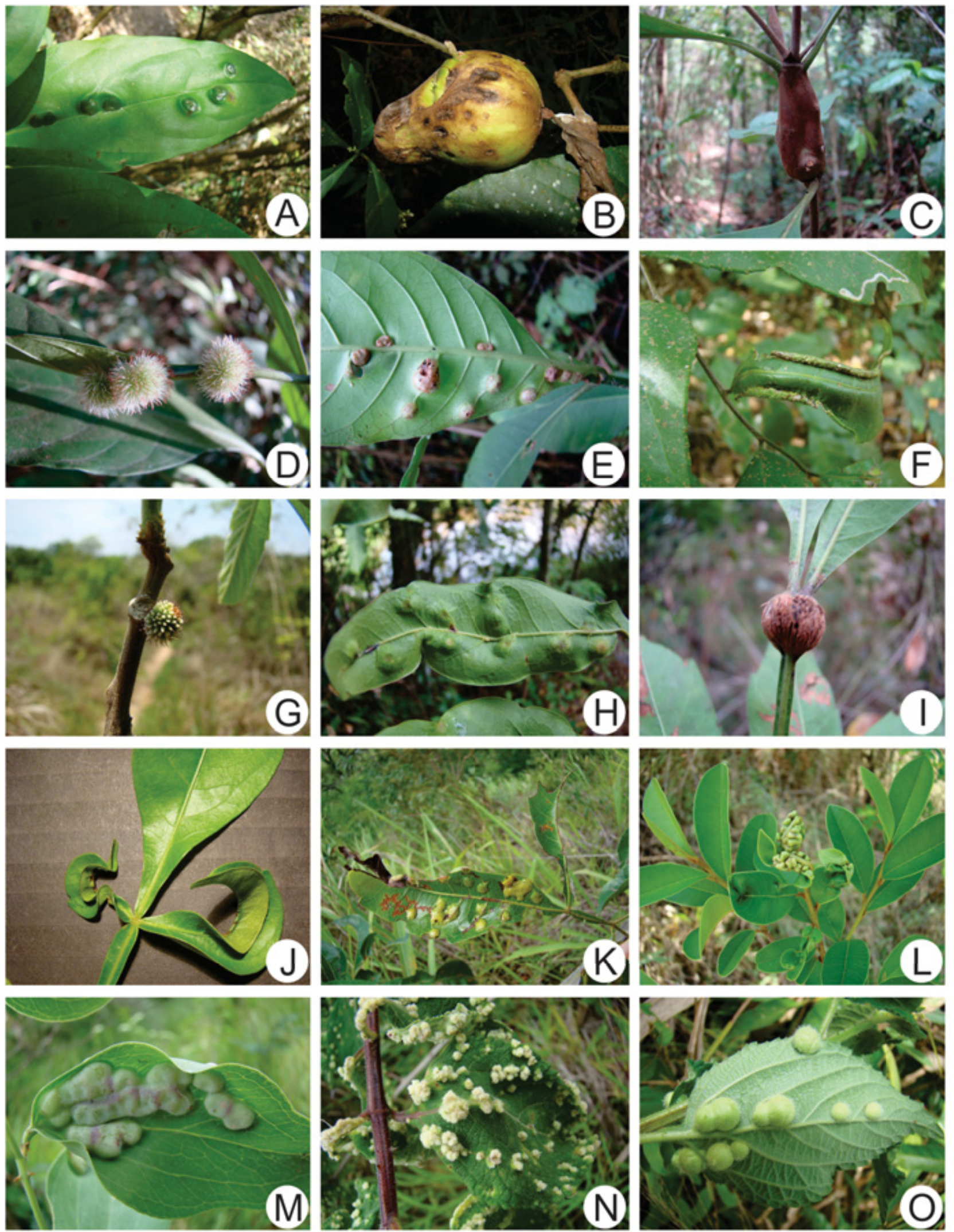

Figure 6. Morphospecies of galls of the ecological trails of the PESV. A-B) Piper arboreum (Piperaceae), C-D) Psychotria carthagenensis (Rubiaceae), E) Psychotria sp. (Rubiaceae), F) Casearia gossypiosperma (Salicaceae), G) Allophylus sericeus (Sapindaceae), H) Matayba sp (Sapindaceae), I-K) Serjania sp (Sapindaceae), L) Chrysophyllum marginatum (Sapotaceae), M) Smilax sp (Smilacaceae), N-O) Lantana camara (Verbenaceae). 
Table 1. Galls of the ecological trails of Parque Estadual Serra Verde. Host plants, gall morphology and associated organisms. 1c: one larval chamber; Mc: more than one larval chamber; grouped: galls can be grouped; midrib: occurs in the midrib of the leaf; petiole: occurs in the petiole of the leaf.

\begin{tabular}{|c|c|c|c|c|c|c|}
\hline \multicolumn{7}{|c|}{ Morphology of the galls } \\
\hline Host Plant & Organ & Morphotype & Color & Other traits & Associated guild & Fig. \\
\hline \multicolumn{7}{|l|}{ Anacardiaceae } \\
\hline Astronium fraxinifolium Schott. & leaf & marginal roll & green & & & $2 \mathrm{~A}$ \\
\hline \multicolumn{7}{|l|}{ Annonaceae } \\
\hline Guatteria sellowiana Schltdl. & leaf & globoid & green & hairy & & $2 \mathrm{~B}$ \\
\hline \multicolumn{7}{|l|}{ Asteraceae } \\
\hline Baccharis dracunculifolia DC. & leaf & leaf fold & green & glabrous; 1c & $\begin{array}{l}\text { Baccharopelma dracunculifoliae } \\
\text { (Hemiptera: Psylidae) }\end{array}$ & $2 \mathrm{C}$ \\
\hline \multirow[t]{2}{*}{ Vernonantura phosphorica Vell. (H. Rob.) } & stem & globoid & brown & glabrous; $1 \mathrm{c}$ & & $2 \mathrm{D}$ \\
\hline & stem & globoid & brown & glabrous & $\begin{array}{c}\text { Tomoplagia rudolphi (Diptera: } \\
\text { Tephritidae) }\end{array}$ & $2 \mathrm{E}$ \\
\hline \multicolumn{7}{|l|}{ Bignoniaceae } \\
\hline Handroanthus ochraceus (Cham.) Mattos & leaf & marginal roll & green & hairy & Thysanoptera; Hemiptera & $2 \mathrm{~F}$ \\
\hline \multicolumn{7}{|l|}{ Boraginaceae } \\
\hline Cordia sellowiana Cham. & leaf & globoid & green & hairy; 1c & Diptera: Cecidomyiidae & $2 \mathrm{G}$ \\
\hline Cordia verbenacia DC. & stem & fusiform & brown & glabrous; $1 \mathrm{c}$ & $\begin{array}{l}\text { Hymenoptera: Eulophidae; } \\
\text { Diptera: Cecidomyiidae }\end{array}$ & $2 \mathrm{H}$ \\
\hline \multicolumn{7}{|l|}{ Burseraceae } \\
\hline \multirow[t]{2}{*}{ Protium heptaphyllum (Aubl.) Marchand } & leaf & lenticular & green & glabrous & & 2I \\
\hline & leaf & globoid & $\begin{array}{l}\text { green / } \\
\text { brown }\end{array}$ & hairy; 1c & Diptera: Cecidomyiidae & $2 J$ \\
\hline \multicolumn{7}{|l|}{ Cannabaceae } \\
\hline \multirow[t]{4}{*}{ Celtis. pubescens (Kunth) Spreng. } & stem & fusiform & brown & glabrous; 1c & $\begin{array}{l}\text { Diptera: Cecidomyiidae; } \\
\text { Hymenoptera }\end{array}$ & $2 \mathrm{~K}$ \\
\hline & stem & conical & green & $\begin{array}{l}\text { grouped; } \\
\text { hairy }\end{array}$ & Diptera: Cecidomyiidae & $2 \mathrm{~L}$ \\
\hline & leaf & globoid & green & $1 \mathrm{c}$ & Diptera: Cecidomyiidae & $2 \mathrm{M}$ \\
\hline & stem & conical & brown & glabrous; 1c & Diptera: Cecidomyiidae & $2 \mathrm{~N}$ \\
\hline \multicolumn{7}{|l|}{ Celastraceae } \\
\hline Maytenus salicifolia Reissek & leaf and stem & globoid & $\begin{array}{l}\text { green e } \\
\text { yellow }\end{array}$ & $\begin{array}{l}\text { glabrous; } \\
\text { adaxial }\end{array}$ & Hymenoptera & $2 \mathrm{O}$ \\
\hline \multicolumn{7}{|l|}{ Erythroxylaceae } \\
\hline Erythroxylum cuneifolium (Mart.) O. E. Schulz. & stem & fusiform & brown & glabrous; $1 \mathrm{c}$ & Hymenoptera & $3 \mathrm{~A}$ \\
\hline Erythroxylum suberosum A.St.-Hil. & leaf & globoid & brown & hairy, 1c & $\begin{array}{c}\text { Dasineura sp. } \\
\text { (Diptera: Cecidomyiidae) }\end{array}$ & 3B \\
\hline \multicolumn{7}{|l|}{ Fabaceae } \\
\hline \multirow[t]{3}{*}{ Bauhinia ungulata $\mathrm{L}$. } & stem & fusiform & brown & glabrous & Diptera: Cecidomyiidae & $3 \mathrm{C}$ \\
\hline & leaf & leaf fold & green & hairy, 1c & Hymenoptera; Lepidoptera & $3 \mathrm{D}$ \\
\hline & leaf & lenticular & green & $\begin{array}{l}\text { grouped; } \\
\text { midrib; } \\
\text { glabrous }\end{array}$ & $\begin{array}{l}\text { Diptera: Cecidomyiidae; } \\
\text { Hymenoptera: Pteromalidae }\end{array}$ & $3 \mathrm{E}$ \\
\hline \multirow[t]{5}{*}{ Copaifera langsdorffii Desf. } & leaf & leaf fold & red / green & hairy; $1 \mathrm{c}$ & Diptera: Cecidomyiidae & $3 \mathrm{~F}$ \\
\hline & leaf & globoid & green & $\begin{array}{l}\text { 1c; cup- } \\
\text { shaped; } \\
\text { glabrous }\end{array}$ & & $3 \mathrm{G}$ \\
\hline & leaf & globoid & red / green & glabrous & Diptera: Cecidomyiidae & $3 \mathrm{H}$ \\
\hline & stem & fusiform & brown & glabrous & Diptera: Cecidomyiidae & $3 \mathrm{I}$ \\
\hline & leaf & fusiform & green & $\begin{array}{l}\text { midrib; } \\
\text { glabrous }\end{array}$ & & $3 \mathrm{~J}$ \\
\hline
\end{tabular}


Table1 . Continuation.

\begin{tabular}{|c|c|c|c|c|c|c|}
\hline \multicolumn{7}{|c|}{ Morphology of the galls } \\
\hline Host Plant & Organ & Morphotype & Color & Other traits & Associated guild & Fig \\
\hline Dalbergia villosa (Benth.) Benth. & stem & fusiform & green & midrib & & $3 \mathrm{~K}$ \\
\hline \multirow[t]{2}{*}{ Inga ingoides Rich (Willd.) } & leaf & globoid & red & abaxial & $\begin{array}{l}\text { Diptera: Cecidomyiidae; } \\
\text { Hymenoptera }\end{array}$ & $3 \mathrm{~L}$ \\
\hline & leaf & globoid & $\begin{array}{l}\text { green / } \\
\text { yellow }\end{array}$ & adaxial & Hymenoptera; Thysanoptera & $3 \mathrm{M}$ \\
\hline $\begin{array}{l}\text { Lonchocarpus cultratus (Vell.) A.M.G. Azevedo } \\
\text { \& H.C. lima }\end{array}$ & leaf & cylindrical & $\begin{array}{l}\text { green / } \\
\text { brown }\end{array}$ & glabrous; 1c & Diptera: Cecidomyiidae & $3 \mathrm{~N}$ \\
\hline Lonchocarpus muehlbergianus Hassl. & leaf & $\begin{array}{l}\text { bivalve } \\
\text { shaped }\end{array}$ & green & $\begin{array}{l}\text { hairy; 1c; } \\
\text { adaxial }\end{array}$ & $\begin{array}{l}\text { Euphalerus ostreoides } \\
\text { (Hemiptera: Psyllidae) }\end{array}$ & 30 \\
\hline Machaerium dimorphandrum Hoehne & leaf & leaf fold & green & $1 \mathrm{c}$ & $\begin{array}{l}\text { Clinodiplosis sp. (Diptera: } \\
\text { Cecidomyiidae) }\end{array}$ & $4 \mathrm{~A}$ \\
\hline \multirow[t]{4}{*}{ Machaerium nyctitans (Vell.) Benth. } & stem & globoid & brown & glabrous; 1c & Hymenoptera & $4 \mathrm{~B}$ \\
\hline & leaf & globoid & green & abaxial & & $4 \mathrm{C}$ \\
\hline & leaf & fusiform & green & $\begin{array}{l}\text { hairy, } 1 c \text {, } \\
\text { midrib }\end{array}$ & Diptera: Cecidomyiidae & $4 \mathrm{D}$ \\
\hline & leaf & globoid & green & $\begin{array}{l}\text { hairy, } 1 \mathrm{c}, \\
\text { adaxial }\end{array}$ & Diptera: Cecidomyiidae & $4 \mathrm{E}$ \\
\hline \multirow[t]{3}{*}{ Piptadenia gonoacantha (Mart.) J.F.Macbr. } & leaf & lenticular & yellow / red & hairy; $1 \mathrm{c}$ & Diptera: Cecidomyiidae & $4 \mathrm{~F}$ \\
\hline & leaf & globoid & green / red & hairy; $1 \mathrm{c}$ & Diptera: Cecidomyiidae & $4 \mathrm{G}$ \\
\hline & leaf & fusiform & brown & rachis & & $4 \mathrm{H}$ \\
\hline Platypodium ellegans Vogel & leaf & leaf fold & green & $1 \mathrm{c}$ & & $4 \mathrm{I}$ \\
\hline \multicolumn{7}{|l|}{ Lauraceae } \\
\hline Nectandra oppositifolia Nees & leaf & marginal roll & green & & & $4 \mathrm{~J}$ \\
\hline \multicolumn{7}{|l|}{ Malpighiaceae } \\
\hline \multirow[t]{3}{*}{ Byrsonima sp. Rich. Ex Kunth. } & stem & amorphous & brown & gouped, hairy & mite & $4 \mathrm{~K}$ \\
\hline & leaf & conical & green & hairy & & $4 \mathrm{~L}$ \\
\hline & leaf & marginal roll & green & hairy & Thysanoptera & $4 \mathrm{M}$ \\
\hline \multicolumn{7}{|l|}{ Malvaceae } \\
\hline \multirow[t]{3}{*}{ Luehea candicans Mart. \& Zucc. } & leaf & globoid & green & hairy, 1c & & $4 \mathrm{~N}$ \\
\hline & leaf & globoid & green & $\begin{array}{l}\text { hairy; 1c; } \\
\text { abaxial }\end{array}$ & & 40 \\
\hline & leaf & lenticular & green & $\begin{array}{l}\text { hairy; } \\
\text { grouped; } \\
\text { midrib }\end{array}$ & & $5 \mathrm{~A}$ \\
\hline \multicolumn{7}{|l|}{ Melastomataceae } \\
\hline Miconia albicans (Sw.) Triana & $\begin{array}{l}\text { leaf / stem / } \\
\text { inflorescence }\end{array}$ & amorphous & green & $\begin{array}{l}\text { grouped; } \\
\text { glabrous }\end{array}$ & $\begin{array}{l}\text { Lepidoptera; Coleoptera; } \\
\text { hymenoptera; spider; } \\
\text { Nematode. }\end{array}$ & $5 \mathrm{~B}$ \\
\hline Miconia ibaguensis (Bonpl.) Triana & $\begin{array}{l}\text { leaf / stem / } \\
\text { inflorescence }\end{array}$ & amorphous & green & $\begin{array}{l}\text { grouped; } \\
\text { glabrous }\end{array}$ & Nematode & 50 \\
\hline \multicolumn{7}{|l|}{ Meliaceae } \\
\hline Trichilia lepdota Mart. & leaf & leaf fold & yellow & hairy; $1 \mathrm{c}$ & & $5 \mathrm{D}$ \\
\hline \multicolumn{7}{|l|}{ Menispermaceae } \\
\hline Abuta sp. Barrère ex Aubl. & stem & fusiform & brown & hairy; $1 \mathrm{c}$ & & $5 \mathrm{E}$ \\
\hline
\end{tabular}


Table1 . Continuation

\begin{tabular}{|c|c|c|c|c|c|c|}
\hline \multicolumn{7}{|c|}{ Morphology of the galls } \\
\hline Host Plant & Organ & Morphotype & Color & Other traits & Associated guild & Fig. \\
\hline \multicolumn{7}{|l|}{ Myrtaceae } \\
\hline Campomanesia sp. Ruiz \& Pav. & stem & fusiform & brown & $\begin{array}{l}\text { hairy; } \\
\text { grouped }\end{array}$ & & $5 \mathrm{~F}$ \\
\hline \multirow[t]{3}{*}{ Eugenia florida DC. } & leaf & conical & white / red & $\begin{array}{l}\text { glabrous; } \\
\text { mc; adaxial }\end{array}$ & $\begin{array}{l}\text { Bruggmannia sp. (Diptera: } \\
\text { Cecidomyiidae) }\end{array}$ & $5 \mathrm{G}$ \\
\hline & leaf & marginal roll & green & glabrous & Hemiptera / Thysanoptera & $5 \mathrm{H}$ \\
\hline & leaf & globoid & green & & Hemiptera & $5 \mathrm{I}$ \\
\hline \multirow[t]{4}{*}{ Myrcia splendens (Sw.) DC. } & bud & $\begin{array}{l}\text { pocket } \\
\text { shaped }\end{array}$ & red & glabrous; 1c & Thysanoptera & $5 \mathrm{~J}$ \\
\hline & leaf & globoid & green & glabrous & Hymenoptera & $5 \mathrm{~K}$ \\
\hline & leaf & marginal roll & green & glabrous & $\begin{array}{l}\text { Holopothrips sp. e } \\
\text { Ramphothrips sp. } \\
\text { (Thysanoptera) }\end{array}$ & $5 \mathrm{~L}$ \\
\hline & stem & fusiform & brown & glabrous & Diptera: Cecidomyiidae & $5 \mathrm{M}$ \\
\hline Myrcia tomentosa (Aubl.) DC. & stem & $\begin{array}{l}\text { pocket } \\
\text { shaped }\end{array}$ & red & glabrous; 1c & & $5 \mathrm{~N}$ \\
\hline Myrciaria floribunda (H. West ex Wild.) O. Berg & leaf & leaf fold & green & glabrous; 1c & Hemiptera / Thysanoptera & $5 \mathrm{O}$ \\
\hline \multicolumn{7}{|l|}{ Piperaceae } \\
\hline \multirow[t]{2}{*}{ Piper arboreum Aubl. } & leaf & lenticular & green & glabrous & $\begin{array}{l}\text { Diptera: Cecidomyiidae; } \\
\text { Hymenoptera: Eulophidae }\end{array}$ & $6 \mathrm{~A}$ \\
\hline & bud & globoid & green & grouped; $\mathrm{mc}$ & $\begin{array}{l}\text { Zalepidota sp. (Diptera: } \\
\text { Cecidomyiidae); Lepidoptera }\end{array}$ & $6 \mathrm{~B}$ \\
\hline \multicolumn{7}{|l|}{ Rubiaceae } \\
\hline \multirow[t]{2}{*}{ Psychotria carthagenensis Jacq. } & bud & fusiform & brown & & & $6 \mathrm{C}$ \\
\hline & leaf & globoid & green / red & $\begin{array}{l}\text { grouped; } \\
\text { hairy; } 1 \mathrm{c}\end{array}$ & Diptera: Cecidomyiidae & $6 \mathrm{D}$ \\
\hline Psychotria sp1 Juss. & leaf & lenticular & $\begin{array}{l}\text { green / } \\
\text { brown }\end{array}$ & grouped/1c & Diptera: Cecidomyiidae & $6 \mathrm{E}$ \\
\hline \multicolumn{7}{|l|}{ Salicaceae } \\
\hline Casearia gossypiosperma Briq. & leaf & marginal roll & green & & & $6 \mathrm{~F}$ \\
\hline \multicolumn{7}{|l|}{ Sapindaceae } \\
\hline Allophylus sericeus (Cambess.) Radlk. & bud & globoid & green / white & hairy; $1 \mathrm{c}$ & Hymenoptera & $6 \mathrm{G}$ \\
\hline Matayba sp. Aubl. & leaf & lenticular & green & grouped & & $6 \mathrm{H}$ \\
\hline \multirow[t]{3}{*}{ Serjania sp. Mill. } & leaf & globoid & brown & hairy; petiole & & $6 \mathrm{I}$ \\
\hline & leaf & lenticular & $\begin{array}{l}\text { green / } \\
\text { yellow }\end{array}$ & midrib & Diptera: Cecidomyiidae & $6 \mathrm{~J}$ \\
\hline & leaf & lenticular & yellow & & & $6 \mathrm{~K}$ \\
\hline \multicolumn{7}{|l|}{ Sapotaceae } \\
\hline $\begin{array}{l}\text { Chrysophyllum marginatum (Hook. \& Arn.) } \\
\text { Radlk. }\end{array}$ & leaf & $\begin{array}{l}\text { pocket } \\
\text { shaped }\end{array}$ & green & glabrous; 1c & Thysanoptera & $6 \mathrm{~L}$ \\
\hline \multicolumn{7}{|l|}{ Smilacaceae } \\
\hline Smilax sp. L. & leaf & globoid & green & grouped & Diptera: Cecidomyiidae & $6 \mathrm{M}$ \\
\hline \multicolumn{7}{|l|}{ Verbenaceae } \\
\hline \multirow[t]{2}{*}{ Lantana camara $\mathrm{L}$. } & leaf & $\begin{array}{l}\text { pocket } \\
\text { shaped }\end{array}$ & $\begin{array}{l}\text { green / } \\
\text { yellow }\end{array}$ & $\begin{array}{l}\text { grouped; } \\
\text { hairy, 1c }\end{array}$ & $\begin{array}{l}\text { Aceria lantanae Cook } \\
\text { (Acarina) }\end{array}$ & $6 \mathrm{~N}$ \\
\hline & leaf & globoid & green & $\begin{array}{l}\text { grouped; } \\
\text { hairy; } 1 \mathrm{c}\end{array}$ & $\begin{array}{l}\text { Schismatodiplosis lantanae } \\
\text { (Diptera: Cecidomyiidae) }\end{array}$ & $6 \mathrm{O}$ \\
\hline
\end{tabular}




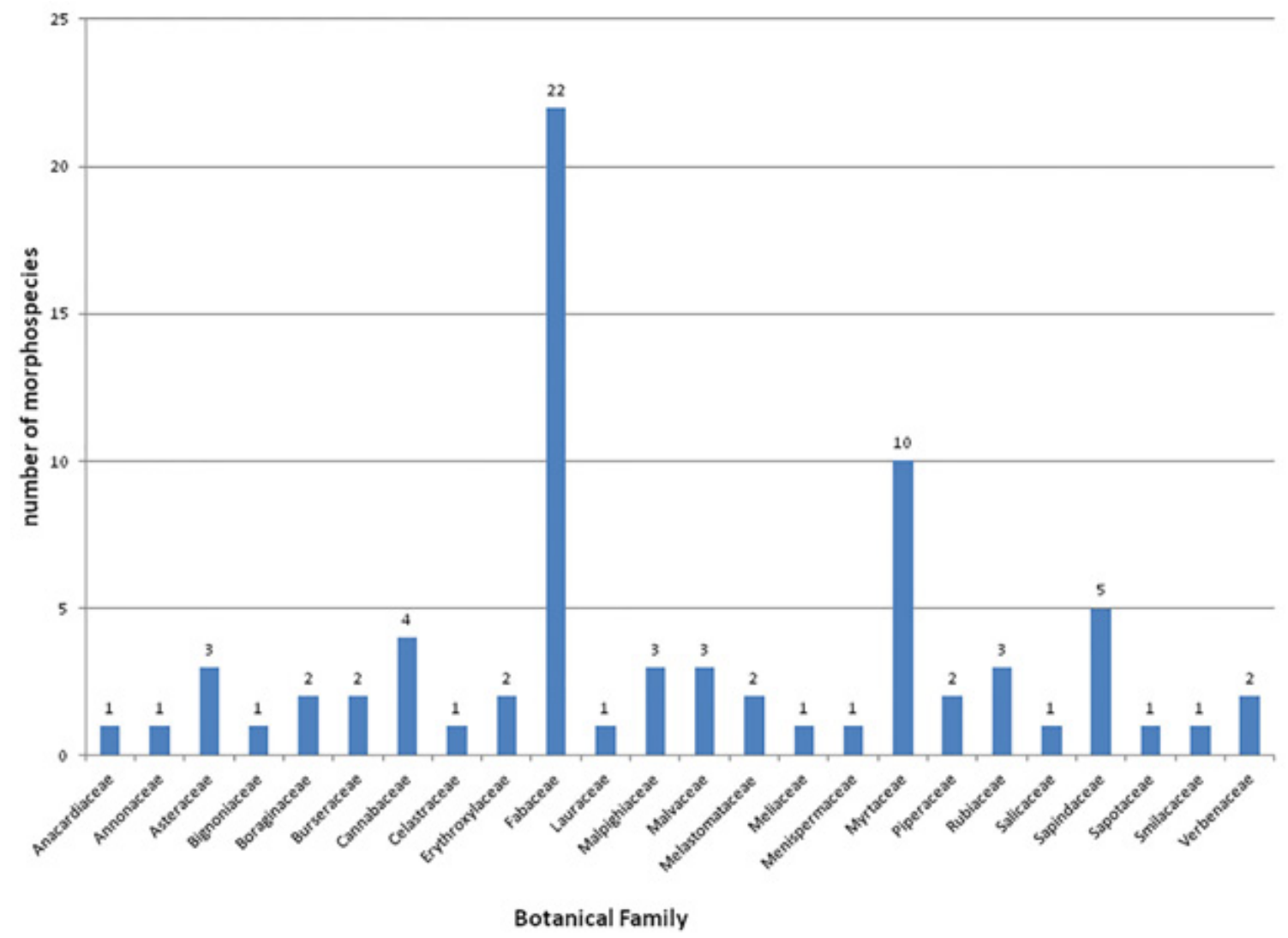

Figure 7. Distribution of morphospecies of galls by their host plant families.

of galls was observed. The host plant species with the most homogeneous temporal distribution were Celtis pubescens (Cannabaceae), Myrcia splendens (Myrtaceae), Lantana camara (Verbenaceae), Luehea candicans (Malvaceae), Byrsonima sp. (Malpighiaceae), Trichilia lepidota (Meliaceae), Miconia albicans, and Miconia ibaguensis (Melastomataceae). It is noteworthy that only four gall morphospecies were detected exclusively within one month, May (in Bauhinia ungulata), August (in Copaifera langsdorffii) and October (in Platypodium ellegans); all Fabaceae hosts.

The spatial distribution of gall morphospecies among the surveyed trails showed that the Trilha do Estacionamento had the lowest frequency (27\%). Only seven plant species were sampled in at least three of the four trails: Vernonanthura phosphorica (Asteraceae), Handroanthus ochraceus (Bignoniaceae), Celtis pubescens (Cannabaceae); Myrcia splendens (Myrtaceae), Abuta sp. (Menispermaceae), and Lantana camara (Verbenaceae). Most morphospecies $(68 \%)$ occurred in only one trail.

Galls were observed within four different plant organs: leaves $(71 \%)$, stems $(21 \%)$, buds (5\%), and inflorescences (3\%). Among the morphotypes suggested by Isaias et al. (2013), the globoid was the most frequent (35\%), followed by the fusiform (17\%), the lenticular (12\%), the marginal roll (9\%), and leaf fold (9\%). The less common morphotypes were the bivalve shaped (1\%) and the cylindrical (1\%). In regard to color, there was prevalence of green $(58 \%)$ and brown $(22 \%)$ galls.

Plant species that hosted the highest number of galls were Copaifera langsdorffii (Fabaceae) containing five morphospecies, and Celtis pubescens (Cannabaceae), Myrcia splendens (Myrtaceae), Machaerium nyctitans (Fabaceae) and Serjania sp. (Sapindaceae) with four morphospecies each. Luehea candicans (Malvaceae), Bauhinia ungulata (Fabaceae), Piptadenia gonoacantha (Fabaceae), Byrsonima sp (Malpighiaceae), and Eugenia florida (Myrtaceae) had three different morphospecies, each.

\section{Discussion}

The plant families with the largest number of species in a given area are usually those which also host a higher richness of galls. The Myrtaceae and the Fabaceae have the highest species richness at Parque Estadual Serra Verde. They were also the main hosts of galls, which confirm the pattern presented in the inventories in the Neotropical areas. This pattern was evidenced at several places in Serra do Espinhaço (Carneiro et al. 2009). Even though the Asteraceae and the Melastomataceae tend to host most of the galls within several surveyed areas (Gonçalves-Alvim 


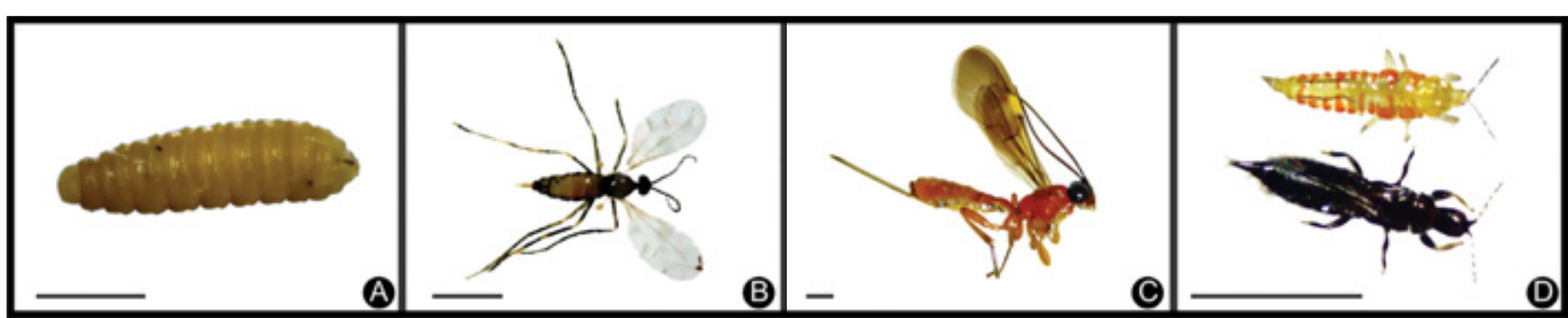

Figure 8. Main groups of galling insects collected at the PESV. a) Diptera: Cecidomyiidae larvae found in gall of Machaerium nyctitans; b) Diptera: Cecidomyiidae adult found in gall of Piper arboreum; c) Hymenoptera found in gall of Bauhinia ungulata; d) Thysanoptera found in gall of Myrciaria floribunda. $($ bar $=1 \mathrm{~mm}$ )

\& Fernandes 2001), they were not widespread at the PESV, with only two species surveyed per family (IEF 2010). Celtis pubescens, the only species of the Cannabaceae occurring in the PESV, hosted up to four morphotypes, which could be attributed to its temporal and spatial distribution in the area.

Another trend observed in the study was the high diversity and richness of the Diptera: Cecidomyiidae and Thysanoptera, as the main inducers of the recorded morphospecies. This data corroborates most of the inventories on the diversity and richness of galls (Espírito-Santo \& Fernandes 2007, Moreira et al. 2007, Coelho et al. 2009, Toma \& Mendonça-Junior 2013). As these inducers are usually found to be new species to science, the PESV seems to be a good area for further studies on insect taxonomy.

The galls induced by these insects had specific distribution along the trails. It can be explained by the trails' peculiarities such as vegetation type, their length, and level of disturbance. The Trilha do Curiango, the longest one (731 meters), and the Trilha do Pica-Pau (456 meters) had more gall morphospecies. These two longest trails are also the most similar in floristic composition and levels of environment impact, such as those caused by fire, human activity and soil erosion; the latter being due mainly to the absence of vegetation cover in particular sections. Likewise, the more homogeneous and dense vegetation of the shorter trails hosts a reduced richness of galls due to the lack of potential host plants.

Similar to other inventories carried out in the Neotropics (Fernandes \& Negreiros 2006, Toma \& Mendonça-Junior 2013), there is a predominance of leaf galls at the PESV. Leaves are usually the most available plant organs and therefore the most susceptible to gall induction. These organs are also more abundant and easily observed, in contrast to the other plant organs; they are more plastic (Valladares et al. 2006, Formiga et al. 2013), and have a larger potential to respond to galling stimuli.

Most recorded galls are greenish in color. In some reported cases, there was a graduation from green to red, brown or yellow through shades of orange and pink. This color variation has also been observed by Fernandes, Neto and Martins (1988), Inbar et al. (2010), and Dias et al. (2013). The green color of the galls reveals the existence of photosynthetic cells, which could be an advantage for host plants, because they increase the photosynthetic surface due to hyperplasia and hypertrophy of plant cells. These green galls may be good models for the study of photosynthesis and the cytological responses to galling stress, as recently investigated by Oliveira et al. (2011). Morever, some studies in tropical areas have demonstrated that the photosynthesis in galls is not enough for the maintenance of their structure, and are employed as an accessory to the host plant machinery, instead (Oliveira et al. 2011, Castro et al. 2012). It has also been proven that the change of colors of the galls may be of aposematic nature to the gall system, as reported for Psidium myrtoides-Nothotrioza myrtoides (Carneiro et al. 2013); it can also be related to the presence or absence of gall inducers, inquilines and parasitoids, as discovered by Dias et al. (2013) for Schinus polygamous-Calophya duvauae. Thus, color variation is another potential field of research to be explored in the galls of the PESV.

Despite some previous studies at similar areas have found the lenticular morphotype (also referred as discoid) as the most abundant morphotype in nature (Fernandes \& Negreiros 2006, Araújo et al. 2007), in the PESV the prevalence was of the globoid and fusiform galls as pointed out by Isaias et al. (2013) in other nine inventories of Brazilian galls (Maia \& Fernandes 2004, Maia et al. 2008, Carneiro et al. 2009, Maia \& Oliveira 2010, Maia 2012, Santos, AlmeidaCortez \& Fernandes 2011a,b, Malves \& Frieiro-Costa 2012, Santos, Almeida-Cortez \& Fernandes 2012). There was a direct relationship between these two shapes and the host organs. Most globoid galls were found on the leaves and the fusiform ones on branches and stems of the host plants. These new biomechanical designs may develop due to the morphogenetic constraints of their host organs, with the fusiform morphotypes obeying the elongation axis of the host's stems. The globoid morphotype should be representative of a developmental process equally distributed in all directions, with the blockage of the laminar shape. This morphotype also represents the shape which has the maximum and most efficient use of superficial area and total volume. These predominant gall shapes may confer best support, anchorage, and effective protection against environmental stresses.

The relationship of these shapes and how costly and/ or effective they are for the host plants and galls alike have 
not been explored yet in any host plant-galling herbivore system. This interaction could be interpreted as an adaptive response or a consequence of the plant physiology associated with the galling stress. Read and Stokes (2006) have proposed that the mechanics design from the scale of cellular anatomy to whole-plant architecture, is fundamental to plant survival but their properties are not easily measured, interpreted or predicted. Plant tissues are known to be more complex than most engineering structures, as they can timely respond to temporal and spatial fluctuations stresses through adjustment in their shape and structure (phenotypic plasticity). Thus, further studies of the forces that lead to the breaking of the patterns of plant response are required to be continued. These studies could than reveal how stressful this interaction can be for both organisms' life span: the host plant and the gall inducers.

This current study demonstrates that the richness of galls and hence of their inducing agents at the PESV are high, even considering that this area is influenced by human interference, with a history of degradation, and loss of natural resources. A survey conducted in an area with a high level of environmental degradation and low botanical diversity, with a sampling effort similar to this study (60h), reported only 29 morphospecies (Fernandes \& Negreiros 2006). Accordingly, at the Estação Ecológica da Universidade Federal de Minas Gerais, a fragment of urban vegetation in the same city of this study (i.e. Belo Horizonte), only 37 morphotypes were identified (Fernandes et al. 1988). On the other hand, in no urban areas such as Serra do Cipó and Serra de São José, which are conservation units with high biodiversity 92 and 137 species of galls, respectively, were found (Coelho et al. 2009, Maia \& Fernandes 2004).

Urban areas can be subject to elevated temperatures, soil compaction, nutrient deficiencies, water stress and invasion of exotic species; these are some of many other factors that do increase the expose of plants to high levels of herbivory (Fernandes et al. 2009 Julião et al. 2005). These factors affect the availability of nutrients and may potentially low the production of secondary defensive compounds by the plants. Therefore, areas most affected by stresses exhibit more susceptibility to herbivores. On the other hand, Julião et al. (2005) demonstrated that green areas in the urban sites are important for sustaining a greater floristic diversity, which also reflects the diversity of galls. Given the higher biodiversity values of these green areas it is crucial that further studies are carried out and published accordingly to make them accessible to the local and general communities. Thus, the high diversity of galls in the PESV is a reflection of an area with high levels of environmental stress but which has, nevertheless, a moderate botanical diversity. These features make this protected area an important site for the continuous environmental conservation and regeneration. Current results, coupled with the hypothesis that galling insect diversity can be considered good bioindicator of environmental quality, highlight the value of Parque Estadual Serra Verde as an area of great importance for future scientific researches, an area of worth of conservation and most suitable for environmental education. These factors bring many benefits for the local and general communities, resulting, thus, in an improvement of life quality for all in the Greater Belo Horizonte.

\section{Acknowledgments}

We would like to thank Ary Oliveira Filho, Pedro Miranda, Mariana Bünger, Jõao Renato Stehmann and the staff of Systematic Lab of UFMG for the identification of the plants; Valéria Cid Maia and Adriano Cavalleri for the identification of the gall inducers. We appreciate the cooperation of the Plant Anatomy Lab of UFMG, specially the Galls Group, the staff of Parque Estadual Serra Verde and IEF-MG for the field support and the scientific license; Tiago Toma for all the ideas and Renê Carneiro and Afonso Portugal for critical reading and the English revision of the manuscript. This study was supported by FAPEMIG and CNPq.

\section{References}

Araújo, W.S.; Gomes-Klein, V.L. \& Santos, B.B. 2007. Galhas Entomógenas Associadas à Vegetação do Parque Estadual da Serra dos Pireneus, Pirenópolis, Goiás, Brasil. Revista Brasileira de Biociências 5(1): 45-47.

Araújo, W.S.; Santos, B.B., Ferreira, H.D. \& Lousa, T.C. 2007. Ocorrência de Galhas Entomógenas na Vegetação do Campus da UFG, em Goiânia, Goiás. Revista Brasileira de Biociências. 5(1):57-59.

Arduin, M. \& Kraus, J.E. 2001. Anatomia de galhas de ambrosia em folhas de Baccharis concinna e Baccharis dracunculifolia (Asteraceae). Revista Brasileira Botânica. 24(1): 63-72.

Carneiro, M.A.A.; Borges, R.A.X.; Araújo, A.P.A. \& Fernandes, G.W. 2009. Insetos indutores de galhas da porção sul da Cadeia do Espinhaço, Minas Gerais, Brasil. Revista Brasileira de Entomologia, 53(4): 570-592.

Carneiro, R.G.S., Burckhardt, D. \& Isaias, R.M.S. 2013. Biology and systematics of gall-inducing triozids (Hemiptera: Psylloidea) associated with Psidium spp. (Myrtaceae). Zootaxa, 3620 (1): 129-146.

Castro, A.C.; Oliveira, D.C.; Moreira, A.S.F.P.; Lemos-Filho, J.P. \& Isaias; R.M.S. 2012. Source-sink relationship and photosynthesis in the hornshaped gall and its host plant Copaifera lagsdorffii Desf. (Fabaceae). South African Journal of Botany, 83: 121-126.

Chase, M.W. \& Reveal, J.L. 2009. A Phylogenetic classification of the land plants to accompany APG III. Botanical Journal of the Linnean Society, 161: 122-127.

Coelho, M.S.; Almada, E.D.; Fernandes, G.W.; Carneiro, M.A.A.; Santos, R.M.; Quintino, A.V. \& Sanchez-Azofeifa, A. 2009. Gall inducing arthropods from a seasonally dry tropical Forest in Serra do Cipó, Brazil. Revista Brasileira de Entomologia, 53(3): 404-414.

Costa, F.V.; Fagundes, M. \& Neves, F.S. 2010. Arquitetura da planta e diversidade de galhas associadas à Copaifera langsdorffii (Fabaceae). Austral Ecology. 20:9-17.

Dias, G.G; Moreira, G.R.P; Ferreira, B.G. \& Isaias, R.M.S. (2013). Why do the galls induced by Calophya duvauae Scott on Schinus polygamus (Cav.) Cabrera (Anacardiaceae) change colors? Biochemical Systematics and Ecology, 48: 111-122.

Dreger-Jauffret, F.; Shorthouse, J.D. 1992. P. 8-33. In: Shorthouse, J.D., Rohfritsch, O. (Eds.), Biology of Insect-Induced Galls. Oxford University Press, Oxford. 
Espírito-Santo, M.M.; Fernandes, G.W. 2007. How many species of gallinducing insects are there on earth, and where are they? Annals of the Entomolical Society of America, 100(2): 95-99.

Fernandes, G.W. \& Martins, R.P. 1985. As Galhas: tumores de plantas. Ciência Hoje, 4: 59-64.

Fernandes, G.W.; Neto, E.T.; Martins, R.P. 1988. Ocorrência e caracterização de galhas entomógenas na vegetação do Campus Pampulha da Universidade Federal de Minas Gerais. Revista Brasileira de Zoologia, 5(1): 11-29.

Fernandes, G.W. \& Price, P.W. 1988. Biogeographical gradients in galling species richness. Oecologia, 76: 161-167.

Fernandes, G.W. 1992. Plant family size and age effects on insular gallforming species richness. Global Ecology and Biogeography, 2: 71-74.

Fernandes, G.W. \& Price, P.W. 1992. The adaptive significance of insect gall distribution: survivorship of species in xeric and Mesic habitats. Oecologia, 90: 14-20.

Fernandes, G.W.; Paula, A.S. \& Loyola Júnior, R. 1995. Distribuição Diferencial de insetos galhadores entre habitats e seu possível uso como bioindicadores. Vida Silvestre Neotropical, 4(2):133-139.

Fernandes, G.W.; Araújo, R.C.; Araújo, S.C.; Lombardi, J.A.; Paula, A.S.; Loyola Júnior, R. \& Cornelissen, T.G. 1997. Insect Galls from Savanna and Rocky Fields of the Jequitinhonha Valley, Minas Gerais, Brazil. Naturalia, 22: 221-244.

Fernandes, G.W. \& Negreiros, D. 2006. A comunidade de insetos galhadores da RPPN Fazenda Bulcão, Aimorés, Minas Gerais, Brasil. Lundiana, 7(2): 111-120.

Fernandes, S.P.C.; Castelo-Branco, B.P.; Albuquerque, F.A.; Ferreira, A.L.N.; Brito-Ramos, A.B.; Braga, D.V.V. \& Almeida-Cortez, J. 2009. Galhas entomógenas em um fragmento urbano de Mata Atlântica no centro de endemismo de Pernambuco. Revista Brasileira de Biociências, 7(3): 240-244.

Formiga, A.T.; Oliveira, D.C.; Ferreira, B.G; Magalhães, T.A.; Castro, A.C; Fernandes, G.W; Isaias, R.M.S. 2013. The role of pectic composition of cell walls in the determination of the new shape-functional design in galls of Baccharis reticularia (Asteraceae). Protoplasma, 250: 899-908.

Gonçalves-Alvim, S.J. \& Fernandes, G.W. 2001. Comunidades de insetos galhadores (Insecta) em diferentes fisionomias do cerrado em Minas Gerais, Brasil. Revista Brasileira de Zoologia, 18(1): 289-305.

IEF, 2010. Plano de Manejo do Parque Estadual Serra Verde, PESV: Encarte I, Encarte II e Resumo Executivo. Governo de Minas Gerais.

Inbar, M.; Izhaki, I.; Koplovich A.; Lupo, I.; Silanikove, N.; Glasser, T.; Gerchman, Y.; Perevolotsky, A. \& Lev-Yadun, S. (2010). Why do many galls have conspicuous colors? A new hypothesis. Arthropod Plant Interactions, 4 (1):1-6.

Isaias, R.M.S.; Carneiro, R.G.S.; Oliveira, D.C. \& Santos, J.C. (2013). Illustrated and Annotated Checklist of Brazilian Gall Morphotypes. Neotropical Entomology, 42:230-239.

Julião, G.R.; Fernandes, G.W.; Negreiros, D., Bedê, L. \& Araújo, R.C. 2005. Insetos galhadores associados a duas espécies de plantas invasoras de áreas urbanas e peri-urbanas. Revista Brasileira de Entomologia, 49(1): 97-106.

Lara, A.C.F. \& Fernandes, G.W. 1996. The highest diversity of galling insects: Serra do Cipó, Brazil. Biodiversity Letters, 3: 111-114.

Maia, V.C. \& Fernandes, G.W. 2004. Insect galls from Serra de São José (Tiradentes, MG, Brazil). Brazilian Journal of Biology, 64(3A): 423-445.
Maia, V.C.; Magenta, M.A.G. \& Martins, S.E. 2008. Ocorrência e caracterização de galhas de insetos em áreas de restinga de Bertioga (São Paulo, Brasil). Biota Neotropica, 8(1): 167-197.

Maia, V.C. \& Oliveira, K.C. 2010. Galhas de insetos da Reserva Biológica Estadual da Praia do Sul (Ilha Grande, Angra dos Reis, RJ). Biota Neotropica, 10(4): 227-238.

Maia, V.C. 2012. Characterization of insect galls, galls makers, and associated fauna of Platô Bacaba (Porto de Trombetas, Pará, Brazil). Biota Neotropica, 11(4): 1-17.

Malves, K. \& Frieiro-Costa, F.A. 2012. Listo f plants with galls induced by insects from the UNILAVRAS/Boqueirão Biological Reserve, Ingaí, state of Minas Gerais, Brazil. Checklist, 8(3): 426-431.

Mani, M.S. 1964. Ecology of plant galls. Dr. W. Junk Publishers, The Hague.

Moreira, R.G.; Fernandes, G.W.; Almada, E.D. \& Santos, J.C. 2007. Galling insects as bioindicators of land restoration in an area of Brazilian Atlantic Forest. Lundiana, 8(2): 107-112.

Oliveira, D.C.; Isaias, R.M.S.; Moreira, A.S.F.P.; Magalhães, T.A. \& LemosFilho, J.P. 2011. Is the oxidative stress caused by Aspidosperma spp. Galls capable of altering leaf photosynthesis? Plant Science, 180: 489-495.

Price, P.W.; Fernandes, G.W.; Lara, A.C.F. \& Brown, J. 1998. Global patterns in local number of insect galling species. Journal of Biogeography, 25: 581-591.

Read, J. \& Stokes, A. 2006. Plant biomechanics in an ecological context. American Journal of Botany, 93 (10): 1546-1565.

Resende, D.C.; Teixeira, E.C.; Benavides, A.M.; Claro-Júnior, L.H. \& Silveira, C.L. 2002. Diversidade de Galhas em Ambientes de Borda e de Interior da Mata. P. 90-92. In Zuanon, J. \& Venticinque, E., Ecologia da Floresta Amazônica - Curso de Campo, 2ed., PDBFF - INPA.

Santos, B.B.; Ferreira, H.D. \& Araújo, W.S. 2010. Ocorrência e Caracterização de Galhas Entomógenas em uma Área de Floresta Estacional Semidecídua em Goiânia, Goiás, Brasil. Acta Botanica Brasilica, 24(1): 243-249.

Santos, J.C.; Almeida-Cortez, J.S. \& Fernandes, G.W. 2011a. Diversity of gall-inducing insects in the high altitude wetland forests in Pernambuco, Northeast Brasil. Brazilian Journal of Biology, 71(1): 47-56.

Santos, J.C.; Almeida-Cortez, J.S. \& Fernandes, G.W. 2011b. Richness of gall-inducing insects in the tropical dry forest (Caatinga) of Pernambuco. Revista Brasileira de Entomologia, 55(1): 45-54.

Santos, J.C.; Almeida-Cortez, J.S. \& Fernandes, G.W. 2012. Gall-inducing insects from Atlantic forest of Pernambuco, Northeastern Brazil. Biota Neotropica. 12(3): 197-213.

Santos-Mendonça, I.V.; Lucena, M.F.A.; Vasconcelos, S.F. \& AlmeidaCortez, J.S. 2007. Caracterização e distribuição espacial de galhas em Clusia nemorosa G. Mey (Clusiaceae) em uma área de Floresta Atlântica, Igarassu, PE. Lundiana 8(1): 49-52.

Silva, M.H.M; Costa-Filho, L.O.; Oliveira, A.F.M. \& Almeida-Cortez, J.S. 2011. Ocorrência de galhas em espécies de Croton no Parque Nacional Vale do Catimbau (PE). Revista Brasileira de Geografia Física, 3: 510-519.

Toma, T.S.P. \& Mendonça-Júnior, M.S. 2013. Gall-inducing insects of an Araucaria Forest in Southern Brazil. Revista Brasileira de Entomologia, 57(2): 225-233.

Valladares, F.; Sanchez-Gomez, D. \& Zavala, M.A. 2006. Quantitative estimation of phenotypic plasticity: bridging the gap between the evolutionary concept and its ecological. Journal Ecology, 94(6): 1103-1116. 